\title{
REMOÇÃO DE RODAMINA B EM ÁGUAS RESIDUÁRIAS DE TINGIMENTO DE PEDRAS SEMIPRECIOSAS POR ELETRO- FENTON
}

\section{I.M. RODEGHERI, K. ZAMBONIN, B. C. LUDWIG, M. HEMKEMEIER e J. S. PICCIN}

Universidade de Passo Fundo, Faculdade de Engenharia Química

E-mail para contato: marafigor@gmail.com; jefersonpiccin@upf.br

\begin{abstract}
RESUMO - Neste trabalho o processo eletro-Fenton foi utilizado como técnica para remoção do corante Rodamina-B (Ro-B) em efluentes de tingimento de pedras semi-preciosas. O efluente foi colocado em uma indústria local, após a lavagens das pedras tingidas, resultando numa concentração inicial de Ro-B na ordem de 1,94 g/L e DQO de $10 \mathrm{~g} / \mathrm{L}$. Após a caracterização, foi submetido ao processo eletro-Fenton em reator de bancada, resultando em remoções do corante Ro-B entre 26,35 \% a 85,31 \% após 120 min de tratamento. Foi observado que a adição de ar no reator e a redução do $\mathrm{pH}$ facilitou a remoção do corante Ro-B. Já o aumento da concentração de $\mathrm{H}_{2} \mathrm{O}_{2}$ não provocou aumento na velocidade de remoção do corante. Os resultados demonstram que o processo eletro-Fenton é promissor para o pré-tratamento de efluentes de tingimento de pedras contendo Ro-B.
\end{abstract}

\section{INTRODUÇÃO}

O desenvolvimento tecnológico industrial proporciona maior qualidade nos produtos, mas também maior geração de resíduos, o que prejudica a sua degradação natural. Com essa problemática em mente, diversos métodos de tratamento de efluentes são estudados objetivando o lançamento em recursos hídricos de forma ambientalmente adequada ou que possam ser reusados dentro de seus processos produtivos, reduzindo custos e preservando o meio ambiente.

Segundo IBGM (1999), o ramo de gemas e joias no Brasil representa cerca de $35 \%$ da produção mundial de gemas, encontrando-se entre as oito principais províncias gemológicas do mundo. Dentre os processos envolvidos, o de tingimento é o que gera maiores quantidades de efluentes que podem causar impactos ambientais. Nesse processo, as pedras ficam mergulhadas em solução alcoólica do corante por um período de tempo, e posteriormente são lavadas em tanques contendo água e detergente para remover o excesso do corante. Durante a lavagem das pedras, é utilizada água em excesso, produzindo um efluente extremamente tóxico, com alta carga orgânica e coloração extremamente concentrada.

Os corantes são amplamente utilizados em processos industriais, como têxtil, plástico, couro, alimentos, tinturaria, papel, indústria farmacêutica e cosmética. Na indústria de pedras, o corante orgânico Rodamina B é utilizado em soluções alcoólicas de tingimento, para a obtenção da coloração rosa. 
Com o objetivo de clarificar estes efluentes e reduzir a toxicidade inerente dos produtos químicos utilizados, técnicas eficientes e economicamente viáveis vêm sendo estudadas. Os processos oxidativos avançados (POAs) são uma alternativa eficaz na degradação de efluentes com cargas tóxicas, principalmente devido ao alto poder oxidante, capaz de promover a degradação de vários compostos, transformando-os em substâncias inertes como dióxido de carbono e água. (SANTOS, 2012; ZELISNKI 2014; FIOREZE; SANTOS; SCHMACHTENBERG, 2014; MACHADO; STÜLP, 2013).

O processo de Eletro-Fenton consiste na produção de radicais hidroxilas no meio, possibilitando assim a mineralização parcial da matéria orgânica. Nidheesh e Gandhimathi (2014) explicam que, no processo de Eletro-Fenton, é adicionado externamente uma quantidade de íons $\mathrm{Fe}^{2+}$ e $\mathrm{Fe}^{3+}$, que reagem com peróxido de hidrogênio $\left(\mathrm{H}_{2} \mathrm{O}_{2}\right)$, que pode ser adicionado ou produzido na superfície do cátodo (Equação 1) gerando radical hidroxila (OH-) no sistema (Equação 2). Esse processo é mais eficiente que o processo Fenton comum porque há a contínua geração de $\mathrm{H}_{2} \mathrm{O}_{2}$ e eletro regeneração do íon ferroso através da redução do cátodo (Equação 3). Isso mantém a produção contínua de hidroxilas no sistema eletrolítico e aumenta a degradação dos poluentes.

$\mathrm{O}_{2}+2 \mathrm{H}^{+}+2 \mathrm{e}^{-} \longrightarrow \mathrm{H}_{2} \mathrm{O}_{2}$
$\mathrm{Fe}^{2+}+\mathrm{H}_{2} \mathrm{O}_{2} \longrightarrow \mathrm{Fe}^{3+}+2 \mathrm{OH}^{-}$
$\mathrm{Fe}^{3+}+\mathrm{e}^{-} \longrightarrow \mathrm{Fe}^{2+}$

Diversos autores investigaram os efeitos dos parâmetros operacionais em sistemas eletro-Fenton, a fim de verificar a remoção do corante Rodamina B a partir de soluções simuladas, com concentrações na ordem de 10 a $50 \mathrm{mg} / \mathrm{L}$, observando remoções próximas a $100 \%$. Porém, poucos estudos reportam resultados observados a partir de efluente real, quando as concentrações podem ser dezenas a centenas de vezes maiores que as reportadas na literatura. Desta forma, o objetivo deste estudo foi estudar uma metodologia capaz de suprir esta necessidade de forma economicamente e ambientalmente viável, através do sistema eletro-fenton aplicados a efluentes reais do corante orgânico sintético Rodamina B.

\section{MATERIAL E MÉTODOS}

A amostra de efluente bruto, contendo o corante Rodamina B foi coletada em uma empresa situada no município de Soledade/RS, seguindo as orientações da norma da ABNT (1987) NBR 9898/97 e foi armazenado em bombonas de 5 L para posterior análises e ensaios. Para a caracterização, o efluente bruto foi submetido a análises físico-químicas compreendendo os parâmetros de demanda química de oxigênio (DQO), cor e pH.

O corante comercial Rodamina B (CI. 45170; Dinamica, P.A) de teor mínimo de 90\% foi utilizado para a obtenção da curva padrão do corante. A concentração do corante em solução, em função da absorbância, foi obtida através do comprimento de onda de maior absorbância do Ro-B $(555 \mathrm{~nm})$. A curva padrão foi obtida através de leituras da absorbância no comprimento de onda verificado, das diluições feitas a partir de uma solução aquosa padrão contendo $1 \mathrm{~g} / \mathrm{L}$. 


\subsection{Caracterização do reator eletrolítico}

O reator eletrolítico é caracterizado por um conjunto de eletrodos formado por um eletrodo não dissociável, constituído de titânio e revestido por óxido de rutênio $\left(\mathrm{Ti} / \mathrm{RuO}_{2}\right)$ e área útil de $0,004478 \mathrm{~m}^{2}$, utilizado como ânodo e um eletrodo de aço-carbono utilizado como cátodo. Os eletrodos são separados por $10 \mathrm{~mm}$ de distância, mergulhados no efluente de forma vertical e ligados individualmente a um cabo condutor e conectados em uma fonte de corrente contínua. A aeração do meio foi realizada através de uma bomba de aquário.

\subsection{Ensaios de eletro-Fenton}

$250 \mathrm{~mL}$ do efluente, após caracterizado, foram adicionados em um reator com volume útil de $500 \mathrm{~mL}$ e teve seu $\mathrm{pH}$ corrigido com $\mathrm{NaOH}$ ou $\mathrm{HCl} 0,1 \mathrm{~mol} / \mathrm{L}$ quando necessário. Após isso, era adicionado o $\mathrm{H}_{2} \mathrm{O}_{2}$ e $\mathrm{FeSO} 4.7 \mathrm{H}_{2} \mathrm{O}$, na proporção de 5:1 $\left(\mathrm{H}_{2} \mathrm{O}_{2}: \mathrm{Fe}^{2+}\right)$, segundo Nidheesh, Gandhimathi e Sanjini (2014). Em sequência, o sistema era acionado, retirando amostras a cada $30 \mathrm{~min}$ durante $2 \mathrm{~h}$. Durante o processo foi controlado a densidade de corrente e monitorado o $\mathrm{pH}$. Os seguintes fatores de estudo foram avaliados:

a) Efeito da injeção de ar: nestes experimentos o efluente teve seu pH ajustado para 4, a densidade de corrente de $150 \mathrm{~A} / \mathrm{m}^{2}$, e concentração inicial de $\mathrm{H}_{2} \mathrm{O}_{2} 82,5 \mathrm{mmol} / \mathrm{L}$.

b) Efeito da concentração inicial de $\mathrm{H}_{2} \mathrm{O}_{2}$ : nestes experimentos o $\mathrm{pH}$ inicial foi ajustado para 3,0, densidade de corrente $200 \mathrm{~A} / \mathrm{m}^{2}$, com injeção de ar e concentração inicial de $\mathrm{H}_{2} \mathrm{O}_{2}$ de $89,5 \mathrm{~mL} / \mathrm{L}$ e $29,8 \mathrm{mmol} / \mathrm{L}$;

c) Efeito do $\mathrm{pH}$ : Nestes experimentos a densidade de corrente foi ajustada para 100 $\mathrm{A} / \mathrm{m} 2$, a concentração inicial de $\mathrm{H}_{2} \mathrm{O}_{2} 44,8 \mathrm{mmol} / \mathrm{L}$, com injeção de ar, avaliando o pH entre 2,0 e 4,0 .

\section{RESULTADOS E DISCUSSÕES}

\subsection{Caracterização do efluente bruto}

Os resultados da caracterização do efluente bruto são apresentados na Tabela 1.

Tabela 1 - Caracterização efluente bruto

\begin{tabular}{cc}
\hline Análise & Resultados* $^{*}$ \\
\hline Concentração de Rodamina-B $(\mathrm{g} / \mathrm{L})$ & $1,85 \pm 0,06$ \\
Cor (Hazen) & $2693 \pm 116,6$ \\
$\mathrm{pH}$ & $3,3 \pm 0,05$ \\
$\mathrm{DQO}(\mathrm{mg} / \mathrm{L})$ & $10113 \pm 597,8$ \\
\hline
\end{tabular}

*média \pm desvio padrão, $\mathrm{n}=3$

O efluente bruto apresenta uma coloração extremamente escura devido à alta concentração de Rodamina B presente no meio $\left(1,9\right.$ g. $\left.\mathrm{L}^{-1}\right)$ resultante do processo de tingimento das pedras. Devido a adição de detergente e álcool no processo de lavagem das pedras, os valores de carga orgânica também são altos $(10113 \mathrm{mg} / \mathrm{L})$, tornando o efluente extremamente tóxico ao meio aquático caso disposto sem tratamento adequado. $\mathrm{Na}$ literatura, os valores de concentração de corante são muito mais baixos. Rathinam et al (2015), 
realizaram experimentos de eletro-fenton com solução com concentração de corante de $50 \mathrm{mg} / \mathrm{L}$.

\subsection{Ensaios experimentais de eletro-fenton}

No ensaio A foi avaliado a injeção de ar no reator, conforme a Figura 1, onde os valores de remoção de Rodamina B foram superiores quando adicionado ar ao reator. Sem a injeção de ar a máxima remoção chegou a $26 \%$ em 2 horas, enquanto que com ar os valores chegaram a quase $70 \%$ de remoção no mesmo tempo, ou seja, em termos de concentração de Rodamina B, a remoção foi de 1,8 g. $\mathrm{L}^{-1}$ a 0,5 g. $\mathrm{L}^{-1}$. Salvador (2011) explicou que com adição de ar em meio ácido, a produção in situ de $\mathrm{H}_{2} \mathrm{O}_{2}$ é favorecida pois o oxigênio dissolvido em solução é transferido para a superfície do cátodo, sofrendo redução e produzindo $\mathrm{H}_{2} \mathrm{O}_{2}$, e ajudando na mineralização do corante.

Figura 1- Efeito da injeção de ar na remoção do corante Ro-B

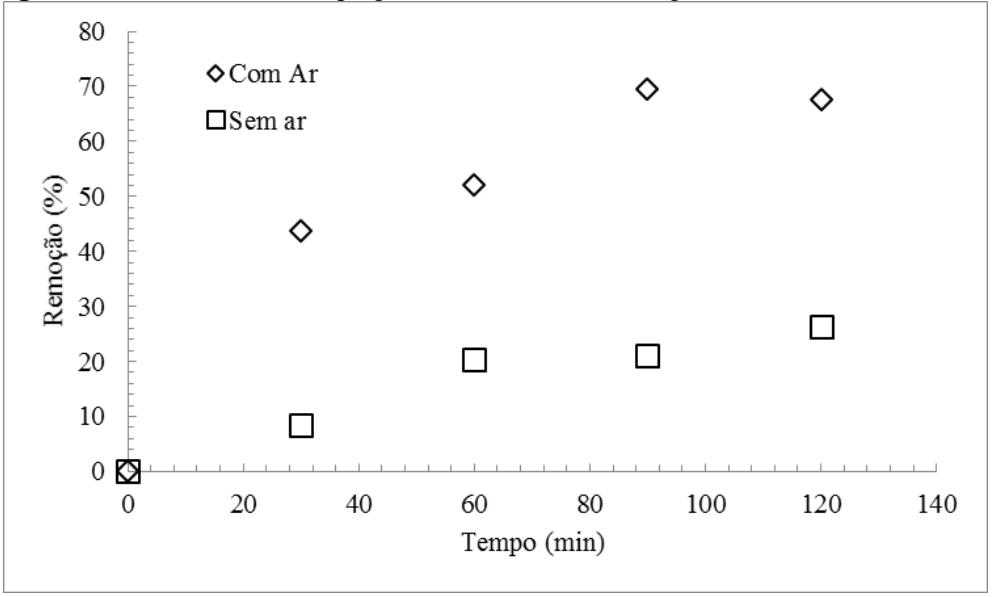

Devido a injeção de ar favorecer o processo e ajudar na remoção de Rodamina B, o ensaio $B$ foi conduzido em novas condições experimentais e o ar foi mantido. Neste experimento foi avaliado a concentração de $\mathrm{H}_{2} \mathrm{O}_{2}$ adicionado ao meio e em 2 horas de tratamento observou-se a mesma remoção, dessa forma a concentração mostra-se não significativa e por isso são necessários novos estudos, conforme Figura 2.

Figura 2- Efeito da concentração de $\mathrm{H}_{2} \mathrm{O}_{2}$ na remoção do corante Ro-B

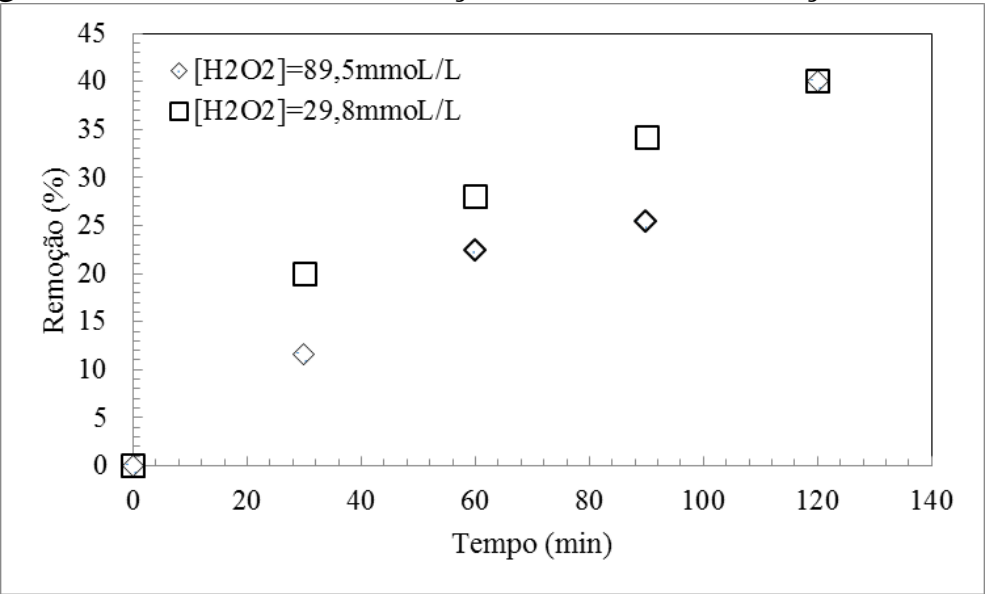


Para avalição do efeito do $\mathrm{pH}$ no processo, novas condições experimentais foram realizadas, porém com valores de $\mathrm{pH}$ diferentes. Os resultados dos efeitos são apresentados na Figura 3 e mostram que em pH 2,0 é de $85 \%$ de remoção de Rodamina B em 2 horas de tratamento, valor superior ao $\mathrm{pH} 4$ em que a máxima remoção foi de $32 \%$. Em relação a concentração do corante em $\mathrm{pH} 2,0$, este variou de 1,3 g.L $\mathrm{L}^{-1}$ para 0,19 g.L $\mathrm{L}^{-1}$. É possível observar a diferença visual entre o efluente bruto e tratado nas Figuras 4 . O efeito de $\mathrm{pH}$ 's mais ácidos são mostrados na literatura como sendo mais favoráveis no processo eletroFenton (Nidheesh, Gandhimathi e Sanjini, 2014), em seus estudos, os autores chegaram à conclusão que o pH igual a 3 é a melhor condição para a produção do radical hidroxila. Segundo Salvador (2011), valores de pH entre 2 e 4, sendo o ideal 3, favorecem a formação de $\mathrm{H}_{2} \mathrm{O}_{2}$, diminuindo a formação de espécies insolúveis de ferro.

Figura 3- Efeito do pH na remoção do corante Ro-B.

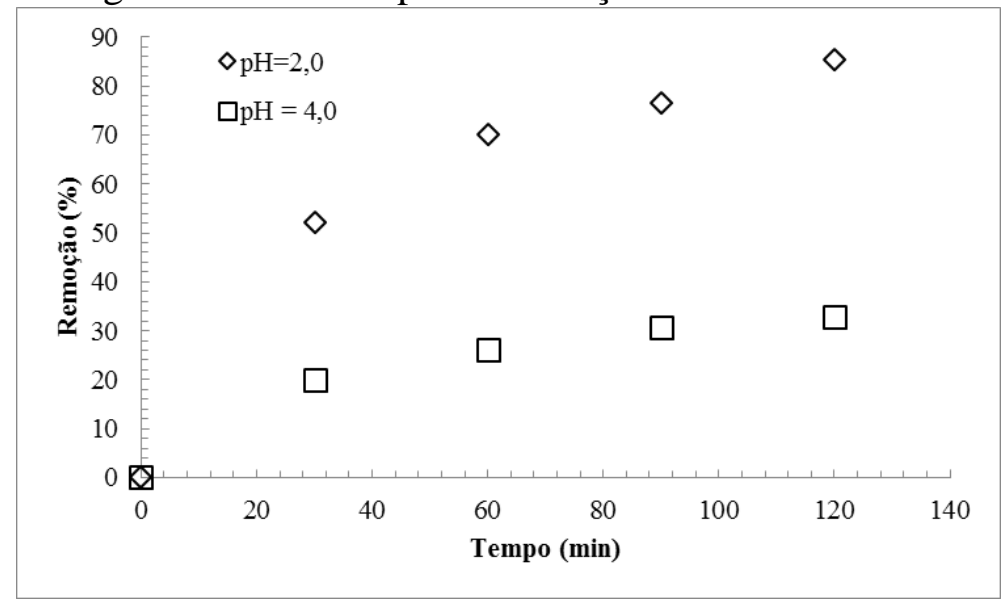

Figura 4- Características visuais do efluente antes (a) e depois do tratamento (b)

a)

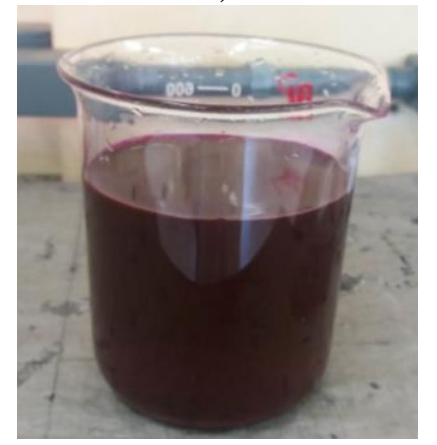

b)

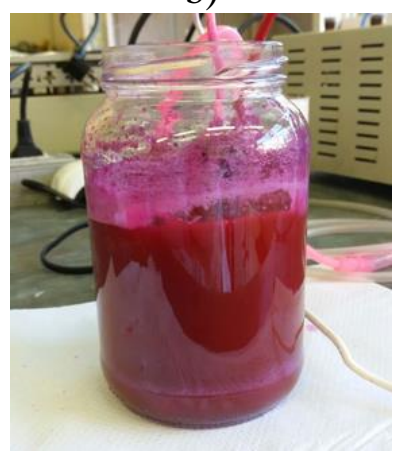

\section{CONCLUSÃO}

Os efeitos dos parâmetros avaliados nos ensaios indicam ótimas remoções de Rodamina B, que chegam a 85\% em pH 2,0 e injeção de ar. Quanto ao efeito do peróxido de hidrogênio este não foi significativo devendo ser estudado posteriormente. Durante os experimentos observou-se a formação de lodo devido ao efluente conter surfactantes, estes adicionados na lavagem das pedras, facilitando a remoção do excesso de corante. 


\section{REFERÊNCIAS}

COSTERNARO, A. Indústria de pedras preciosas: um estudo dos fatores competitivos em empresas de Soledade - RS. Universidade Federal de Santa Maria, RS, 2005.

FIOREZE, M.; SANTOS, E. L.; SCHMACHTENBERG, N., Processos oxidativos avançados: fundamentos e aplicação ambiental. Revista Eletrônica em Gestão, Educação e Tecnologia Ambiental, n. 1, v. 18, p. 79-91, 2014.

MACHADO, V.R STÜLP, S. Avaliação do processo de oxidação em fluxo de efluente sintético proveniente do tingimento de gemas contendo o corante Rodamina B. CeteclUnivates, v. 2, n. 4, p. 45-57, 2010.

MAHARANA, D et al. Eletrochemical degradation of Rhodamine B over $\mathrm{Ti} / \mathrm{SnO}_{2}-\mathrm{Sb}$ Electrode, Water Enviroment Research Beijing Normal University, Pequim, v. 87, n. 4, abr. 2015.

NIDHEESH, P.V, G GANDHIMATHI, R, SANJINI, N.S. Remoção de Rodamina B a partir de solução aquosa com eletrodos de grafite e sistema eletro Fenton. Departament of Civil Engineering, National Institute of Technology, Tiruchirappalli, Tamilnadu, Índia, v.1, n.1 ago. 2014.

NIDHEESH, P.V, GANDHIMATHI, R. Electro Fenton oxidation for the removal of Rhodamine $B$ from aqueous solution in a bubble column reactor under continuous mode. Departament of Civil Engineering, National Institute of Technology, Tiruchirappalli, Tamilnadu, Índia, v.1 n, 1, mar. 2014.

PICCIN, J. S., VIEIRA, M. L. G., GONÇALVES, J., DOTTO, G. L., PINTO, L. A. A., Adsorption of FD\&C Red No. 40 by chitosan: Isotherms analysis. Journal of Food Engineering, 95, 16-20 (2009).

SALVADOR, T. Degradação de corantes têxteis por processos oxidativos avançados do tipo Fenton. Universidade Federal do Paraná, setor de Ciências Exatas, Departamento de Química, Programa de Pós-Graduação em Química. Curitiba - PR, 2011.

RATHINAM, R. et al. (2015): Decolourization of Rhodamine B from aqueous solution by electrochemical oxidation using graphite electrodes, Desalination and Water Treatment, DOI: 10.1080/19443994.2015.1086960

WERMUTH, T. B. et al. Descolorimento de efluente associado ao tingimento de ágatas contendo Rodamina b com diferentes combinações fotocatalíticas via processos oxidativos avançados (poa's) Engenharia Ambienta Universidade de Santa Cruz do Sul, Rio Grande do Sul, v. 17, n. 2, p. 117-123 jul./dez. 2013. Disponível em: <http://online.unisc.brlseerlindex.phpltecn ologia/>. Acesso em: 29 ago. 2016.

ZELINSKI, R. Aplicação de Processos Oxidativos Avançados em Efluentes de Gemas. Centro universitário UNIVATES, Curso de Engenharia Ambiental. Lajeado - RS, 2014. 